\title{
NEW EARTHWORM SPECIES AND RECORDS FROM THE SOUTHERN CARPATHIANS (MEGADRILI: LUMBRICIDAE)
}

\author{
Tímea Szederjesi ${ }^{1}$, Victor V. Pop ${ }^{2}$, Orsolya Márton ${ }^{3}$ and Csaba Csuzdi ${ }^{4}$ \\ ${ }^{1}$ Department of Zoology, Hungarian Natural History Museum, H-1088 Budapest, Baross u. 13, \\ Hungary E-mail: t.szederjesi@gmail.com; https://orcid.org/0000-0001-7695-1468 \\ ${ }^{2}$ Institute of Biological Research, Cluj-Napoca, Romania E-mail: vopop2001@yahoo.com \\ ${ }^{3}$ Institute for Soil Sciences and Agricultural Chemistry, Centre for Agricultural Research, \\ Hungarian Academy of Sciences, Budapest, Hungary E-mail: marton.orsolya@agrar.mta.hu \\ ${ }^{4}$ Department of Zoology, Eszterházy Károly University, Eger, Hungary \\ E-mail: csuzdi.csaba@ektf.hu; https://orcid.org/0000-0002-0319-7836
}

Recent collecting trips to different parts of the Southern Carpathians resulted in reporting 26 earthworm species, among them two species new to science: Dendrobaena cinerea sp. n. and Octodrilus banaticus sp. n. Moreover, Allolobophora pannonica (Cognetti, 1906) has been resurrected from synonymy of $A$. mehadiensis mehadiensis Rosa, 1895 and reinstated as a separate species. With all these, the number of earthworm species and subspecies present in Romania is now 80.

Key words: earthworms, Lumbricidae, new species, Carpathians, Romania.

\section{INTRODUCTION}

The earthworm fauna of Romania is quite well-known. The research began at the end of the 19th century (ÖRLEY 1881, 1885) and continued during the 20th century (Michaelsen 1891, 1903, Černosvitov 1932, Pop 1949, Zicsi \& PoP 1984). In his summarizing work VICTOR Pop (1949) recorded the presence of 47 lumbricid taxa in the country, which was soon complemented to 50 (Рop 1964). As a result of the continuous exploratory work, the number of earthworm species and subspecies has recently reached 71 (Pop et al. 2012). Since then, a new faunistical record and description of five new taxa raised the number of earthworm species present in Romania to 77 (SzEDERJEsi et al. 2014, 2016, 2017).

It is worth mentioning that most of the earlier researches focused mainly on Transylvania and the inner Carpathians, and not much collecting expeditions were led to the outer areas of the mountain range. However, this region harbours a special earthworm fauna with strong East Balkanic affinities (Csuzdi et al. 2011).

To explore these remote regions of the Carpathian chain we have led several expeditions between 2004 and 2009 resulted in recording altogether 41 earthworm taxa including a species new to science and a greyish specimen which phenetically looked similar to the Dendrobaena platyura species group 
but had male pores on segment 15 (SzEDERJEsi et al. 2014). Unfortunately we had only a single specimen of this interesting species therefore in 2016 we have visited the region again but our effort was unsuccessful. However, the first author (TSZ) during a visit to the Natural History Museum, Vienna discovered two well-preserved specimens of this interesting species collected by L. Ganglbauer in Mehadia, Romania. The aim of this paper is to present the results of the last earthworm collecting trips to different parts of the Southern Carpathians, with reinstatement of a formerly synonym species and description of two species new to science including the one which was reported as Dendrobaena sp. in our previous paper (SzEDERJEsI et al. 2014).

\section{MATERIAL AND METHODS}

Earthworms were collected by the diluted formaldehyde method (RAw 1959), complemented with digging and hand-sorting. The specimens were killed and fixed in $96 \%$ ethanol and deposited in the earthworm collection of the Hungarian Natural History Museum (HNHM). Additional material housed in the Natural History Museum Vienna (NHMV) was also examined.

Altogether 16 partial 5.8 rDNA-ITS2-partial 28S rDNA (ITS2) sequences were used for the phylogenetic reconstruction (Table 1). The protocol described by Szederjesi et al.

Table 1. Codes, collecting localities and GenBank accession numbers of the specimens analysed.

\begin{tabular}{lclc}
\hline Species & $\begin{array}{c}\text { HNHM } \\
\text { code }\end{array}$ & Locality & $\begin{array}{c}\text { GenBank } \\
\text { Accession No. }\end{array}$ \\
\hline Dendrobaena cinerea sp. n. & 16810 & Romania: Măru & MH843156 \\
D. byblica & 16160 & Albania: Korab Mts & KT823901 \\
D. byblica & 16214 & Montenegro: Sinjajevina Mts & KT823902 \\
D. byblica & 16457 & Serbia: Golubac & KT823903 \\
D. byblica & 16185 & Greece: Karpathos & KT823898 \\
D. byblica & 16661 & Greece: Crete & KT823899 \\
D. daghestanensis & 16105 & Georgia: Borjomi & KT823900 \\
D. platyura & 16439 & Hungary: Velem & KT823916 \\
D. depressa & 16379 & Bulgaria: Stara Planina & KT823911 \\
D. carpathomontana & 15963 & Romania: Ciumârna & KT823914 \\
D. olympiaca & 16629 & Greece: Naxos & KT823905 \\
D. olympiaca & 15824 & Greece: Sitena & KT823906 \\
D. annectens & 16249 & Romania: Cerna Sat & KT823907 \\
D. transylvanica & 15497 & Romania: Zarand Mts & KT823908 \\
D. herculis & 16231 & Romania: Ponoarele & KT823904 \\
H. mozsaryorum & 16134 & Hungary: Aggtelek karst & KT823922 \\
\hline
\end{tabular}


(2017) was used for obtaining the ITS2 sequence of Dendrobaena sp. n. The new sequence has been uploaded to the GenBank database (BENson et al. 2012). The alignment was performed using the online server of MAFFT v7 (КАтон \& STANDLEy 2013). The phylogenetic tree was constructed using Bayesian analysis. Estimations with MEGA V6.0 using the Bayesian information criterion (BIC) (SchwARz 1978) revealed that the best fitting model for ITS2 was GTR+G. Bayesian analysis was performed with MrBayes 3.2 (RonQuist et al. 2012), using Metropolis Coupled Markov Chain Monte Carlo simulations for 10 million generations, sampling a tree every 1000 generations. After removing the first 2000 trees as burn-in, FigTree 1.4.0 (RAmbaut 2012) was used for visualization.

\section{TAXONOMY}

\section{Allolobophora pannonica (Cognetti, 1906) stat. restit.}

Helodrilus ictericus var. pannonica Cognetti, 1906: 19.

Allolobophora mehadiensis var. pannonica: Pop 1948: 109.

Allolobophora icterica var. pannonica: Pop 1949: 63.

Serbiona pannonica: Mršić 1991: 198.

Allolobophora mehadiensis mehadiensis (part.): Pop et al. 2012: 63.

Material examined. NHMV/5009 1 ex. adult lectotypus +1 ex. juvenile cut specimen, Südost-Siebenbürgen, Paring Gebirg (Southeast Transylvania, Parâng Mts), leg. L. Ganglbauer. HNHM/17191 1 ex., Parâng Mts, N of Novaci, 675 m, 45²12.0436' N $23^{\circ} 41.5426^{\prime} \mathrm{E}$, pasture with alder and hawthorn, leg. Cs. Csuzdi, L. Dányi, V. V. Pop, T. Szederjesi, 12.11.2016. HNHM/17195 1 ex., Parâng Mts, between Novaci and Rânca, 722 m, $45^{\circ} 12.2333^{\prime} \mathrm{N} 23^{\circ} 41.8088^{\prime} \mathrm{E}$, beech forest, leg. Cs. Csuzdi, L. Dányi, V. V. Pop, T. Szederjesi, 12.11.2016.

External characters. Length 63-109 $\mathrm{mm}$ and 4.5-5 mm wide. Number of segments 156-172 with secondary and tertiary annulations. Colour light greyish. Prostomium epilobous $1 / 2$ closed. First dorsal pore at the intersegmental furrow 9/10. Setae closely paired. Setal arrangement behind clitellum: aa:ab:bc:cd: $d d=19.94: 1.51: 11.83: 1: 33.76$. Male pores on segment 15 , surrounded by glandular crescents, confined in its own segment. Nephridial pores invisible. Clitellum on segments $1 / 234,35-46$. Tubercula pubertatis on segments 38-46. Glandular tumescence on segments 13, 15-20, 32-38 ab.

Internal characters. Septa 5/6-9/10 strongly, 10/11-13/14 slightly thickened. Testes and funnels paired in segments 10-11. Two pairs of seminal vesicles in 11-12. Two pairs of spermathecae in 9/10,10/11 with external openings in setal line $d$. Calciferous glands in 10-12 with diverticula in segment 10. Paired hearts in segments 7-11. with a pair of small extraoesophageal vessels in 12. Nephridial bladders J-shaped, proclinate. Crop in segments 15-16, and gizzard in segments 17-18. Typhlosolis multilobous. The cross-section of the longitudinal muscle layer of fasciculated type.

Remarks. Cognetti (1906) described this species as a variety of Aporrectodea icterica (Savigny, 1826) based on a subadult specimen, mentioning only a few characteristics (e.g. the clitellum) and highlighting that all the others are similar to Ap. icterica. Since then the different authors revised this taxon based exclusively on the original description, without examining the type 
Table 2. Distinguishing characters of A. pannonica and the A. mehadiensis subspecies.

\begin{tabular}{|c|c|c|c|c|c|}
\hline Taxon & $\begin{array}{l}\text { Body length } \times \\
\text { width }\end{array}$ & $\begin{array}{l}\text { Segment } \\
\text { number }\end{array}$ & Clitellum & Tubercles & Vesicles \\
\hline $\begin{array}{l}\text { A. pannonica (Cog- } \\
\text { netti, 1906) }\end{array}$ & $\begin{array}{c}63-109 \mathrm{~mm} \times \\
4.5-5 \mathrm{~mm}\end{array}$ & $156-172$ & $1 / 234,35-46$ & $38-46$ & 11,12 \\
\hline $\begin{array}{l}\text { A. mehadiensis meha- } \\
\text { diensis Rosa, } 1895\end{array}$ & $\begin{array}{l}150-210 \mathrm{~mm} \times \\
4-10 \mathrm{~mm}\end{array}$ & $200-270$ & $\begin{array}{c}35,36-47 \\
48\end{array}$ & $41,42-47,48$ & 9-12 \\
\hline $\begin{array}{l}\text { A. mehadiensis } \\
\text { boscaiui Pop, } 1948\end{array}$ & $\begin{array}{l}95-190 \mathrm{~mm} \times \\
6-8 \mathrm{~mm}\end{array}$ & $228-248$ & $\begin{array}{c}33,34-44 \\
45,46\end{array}$ & $38-43,44,45$ & 9-12 \\
\hline $\begin{array}{l}\text { A. mehadiensis oreo- } \\
\text { phila Pop, } 1978\end{array}$ & $\begin{array}{c}143-270 \mathrm{~mm} \times \\
5-8 \mathrm{~mm}\end{array}$ & $260-299$ & $\begin{array}{c}34,35-49 \\
50\end{array}$ & $\begin{array}{c}39,40 \\
41-45,46\end{array}$ & $9-12$ \\
\hline
\end{tabular}

specimens. Pop (1948) while describing A. mehadiensis var. boscaiui, revised the morphologically similar taxa and regarded pannonica as a variety of mehadiensis, basically because it fits well the Dacian mehadiensis group with its type locality in the Parâng Mts, Southern Carpathians, instead of Ap. icterica, which is mostly found in France, Belgium, Switzerland and Italy. Pop also emphasized that even though pannonica has only two pairs of vesicles similarly to Ap. icterica, most $A$. mehadiensis subspecies have very small or reduced vesicles in segment 9 and 10, and here probably a transition can be observed between the 4 pairs type and the 2 pairs.

Mršrć (1991), in his monograph, placed pannonica into the Carpatho-Balkanic genus Serbiona and raised it to species level, but without any remark. On the other hand, Pop et al. (2012) regarded pannonica as a synonym of A. mehadiensis mehadiensis Rosa, 1895 because of the position of its clitellar organs, based on the original description.

Morphologically, some newly collected specimens from the Parâng Mts. showed high similarity with the syntypes housed in the earthworm collection of the Natural History Museum Vienna and revealed just slight differences from the original description, mainly in the position of the tubercles: 38-46 vs. 35-44 (the latter is similar to Ap. icterica). Its body dimensions, the number of vesicles, the position of the clitellum and the tubercles clearly differentiate pannonica from the mehadiensis subspecies (Table 2) and justify its species level status. From the two syntype specimens in the collection of the NHMV, the more or less adult specimen has been selected as lectotype.

\section{Allolobophora robusta Rosa, 1895}

Allolobophora robusta Rosa, 1895: 2. Pop et al. 2012: 63.

Material examined. HNHM/16710 1 ex., Țarcu Mts, beech forest on blacktop $3 \mathrm{~km}$ E of Borlova, 530 m, N45.35276 E22.3900, leg. I. Iorgu, R. Kleukers, B. Odé, G. Puskás, G. 
Szövényi, L. Willemse, 09.07.2013. HNHM/16711 1 ex., Ţarcu Mts, beech-spruce forest, 1.5 km NW of Cuntu hut, 1370 m, N45.30911 E22.48827, leg. I. Iorgu, R. Kleukers, B. Odé, G. Puskás, G. Szövényi, L. Willemse, 09.07.2013. HNHM/17237 4 ex., Ţarcu Mts, between Măru and Poiana Mărului, 443 m, 45 $26.0775^{\prime} \mathrm{N} 22^{\circ} 27.4282^{\prime} \mathrm{E}$, mixed beech forest, leg. Cs. Csuzdi, L. Dányi, V.V. Pop, T. Szederjesi, 10.11.2016.

\section{Aporrectodea caliginosa caliginosa (Savigny, 1826)}

Enterion caliginosum Savigny, 1826: 180.

Aporrectodea caliginosa: Pop et al. 2012: 63.

Material examined. HNHM/17193 4 ex., Parâng Mts, N of Novaci, 675 m, $45^{\circ} 12.0436^{\prime} \mathrm{N} 23^{\circ} 41.5426^{\prime} \mathrm{E}$, pasture with alder and hawthorn, leg. Cs. Csuzdi, L. Dányi, V.V. Pop, T. Szederjesi, 12.11.2016. HNHM/17198 2 ex., Parâng Mts, N of Novaci, bank of Gilort, 658 m, $45^{\circ} 13.388^{\prime} \mathrm{N} 23^{\circ} 40.22^{\prime}$ E, mixed forest, leg. Cs. Csuzdi, L. Dányi, V.V. Pop, T. Szederjesi, 11.11.2016. HNHM/17215 1 ex., Parâng Mts, stream valley NW of Novaci, $505 \mathrm{~m}, 45^{\circ} 11.5962^{\prime} \mathrm{N} 23^{\circ} 39.95^{\prime} \mathrm{E}$, chestnut forest patch, leg. Cs. Csuzdi, L. Dányi, V.V. Pop, T. Szederjesi, 13.11.2016. HNHM/17234 6 ex., Țarcu Mts, S of Măru, 399 m, 45²7.6147'N $22^{\circ} 26.7447^{\prime}$ E, pasture, leg. Cs. Csuzdi, L. Dányi, V.V. Pop, T. Szederjesi, 10.11.2016.

\section{Aporrectodea rosea (Savigny, 1826)}

Enterion roseum Savigny, 1826: 182.

Aporrectodea rosea: Pop et al. 2012: 63.

Material examined. HNHM/17194 1 ex., Parâng Mts, $\mathrm{N}$ of Novaci, 675 m, $45^{\circ} 12.0436^{\prime} \mathrm{N}$ $23^{\circ} 41.5426^{\prime} \mathrm{E}$, pasture with alder and hawthorn, leg. Cs. Csuzdi, L. Dányi, V.V. Pop, T. Szederjesi, 12.11.2016. HNHM/17220 2 ex., Vâlcan Mts, N of Vaidei, bank of Şuşita Verde, 425 $\mathrm{m}, 45^{\circ} 11.6738^{\prime} \mathrm{N} 23^{\circ} 16.1299^{\prime} \mathrm{E}$, beech forest, leg. Cs. Csuzdi, L. Dányi, V.V. Pop, T. Szederjesi, 11.11.2016. HNHM/17235 1 ex., Ţarcu Mts, S of Măru, 399 m, 45 27.6147’N 22²6.7447'E, pasture, leg. Cs. Csuzdi, L. Dányi, V.V. Pop, T. Szederjesi, 10.11.2016. HNHM/17242 2 ex.,

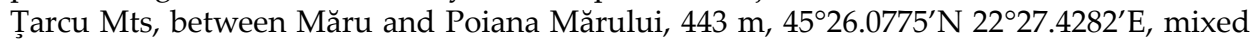
beech forest, leg. Cs. Csuzdi, L. Dányi, V.V. Pop, T. Szederjesi, 10.11.2016.

\section{Bimastos rubidus (Savigny, 1826)}

Enterion rubidum Savigny, 1826: 182.

Dendrodrilus rubidus rubidus: Pop et al. 2012: 63.

Dendrodrilus rubidus subrubicundus: Pop et al. 2012: 63.

Bimastos rubidus: Csuzdi et al. 2017: 20.

Material examined. HNHM/17200 1 ex., Parâng Mts, N of Novaci, bank of Gilort,

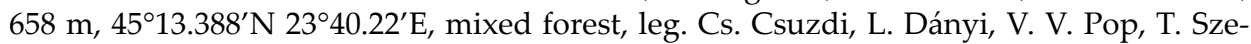
derjesi, 11.11.2016. HNHM/17204 1 ex., Parâng Mts, between Novaci and Rânca, 1132 $\mathrm{m}, 45^{\circ} 13.885^{\prime} \mathrm{N} 23^{\circ} 41.2141^{\prime} \mathrm{E}$, alpine pasture with scattered beech trees, leg. Cs. Csuzdi, L. Dányi, V. V. Pop, T. Szederjesi, 12.11.2016. HNHM/17210 2 ex., between Tismana and Peştişani, $182 \mathrm{~m}, 4^{\circ} 2.62^{\prime} \mathrm{N} 22^{\circ} 59.49^{\prime} \mathrm{E}$, mixed hornbeam and oak forest, leg. Cs. Csuzdi, L. 
Dányi, V. V. Pop, T. Szederjesi, 11.11.2016. HNHM/17217 1 ex., Parâng Mts, stream valley NW of Novaci, $505 \mathrm{~m}, 45^{\circ} 11.5962^{\prime} \mathrm{N} 23^{\circ} 39.95^{\prime} \mathrm{E}$, chestnut forest patch, leg. Cs. Csuzdi, L. Dányi, V. V. Pop, T. Szederjesi, 13.11.2016.

\section{Cernosvitovia rebeli (Rosa, 1897)}

Allolobophora rebelii Rosa, 1897: 2.

Cernosvitovia rebelii: Pop et al. 2012: 63.

Cernosvitovia rebeli: Szederjesi et al. 2014: 91.

Material examined. HNHM/17209 1 ex., between Tismana and Peştişani, $182 \mathrm{~m}$, $45^{\circ} 2.62^{\prime} \mathrm{N} 22^{\circ} 59.49^{\prime} \mathrm{E}$, mixed hornbeam and oak forest, leg. Cs. Csuzdi, L. Dányi, V. V. Pop, T. Szederjesi, 11.11.2016. HNHM/17212 11 ex., between Borlova and Baia de Aramă, 216 m, $45^{\circ} 0.4919^{\prime} \mathrm{N} 22^{\circ} 48.8658^{\prime} \mathrm{E}$, mixed hornbeam and oak forest with beech and chestnut, leg. Cs. Csuzdi, L. Dányi, V. V. Pop, T. Szederjesi, 11.11.2016. HNHM/17229 1 ex., Țarcu Mts, between Borlova and Muntele Mic, $495 \mathrm{~m}, 4^{\circ} 20.5163^{\prime} \mathrm{N} 22^{\circ} 24.4343^{\prime} \mathrm{E}$, mixed beech forest, leg. Cs. Csuzdi, L. Dányi, V. V. Pop, T. Szederjesi, 10.11.2016.

\section{Dendrobaena cinerea Szederjesi, Pop \& Csuzdi sp. n.} (Fig. 1)

Dendrobaena sp.: Szederjesi et al. 2014: 97.

Holotype. HNHM/16810 1 ex., Țarcu Mts, after Măru, beech forest, 501 m, N45²5.978' E22 27.550', leg. Cs. Csuzdi, J. Kontschán, V. V. Pop, 02.11.2007.

Paratypes. NHMV/4990 2 ex., Mehadia, leg. L. Ganglbauer.

Etymology. The name refers to the special colour of the specimens ('cinerea' = greyish).

Diagnosis. Length 113-132 mm, diameter 5-6 mm, setae distantly standing. Pigmentation grey with slight reddish hints. First dorsal pore in 8/9. Clitellum on $25-30$, tubercles on $1 / 2$ 25-1/2 29. Male pores hardly seen on segment 15. Nephridial pores aligned above setal line $b$. Four pairs of seminal vesicles in 9-12; spermathecae 9/10, 10/11 in setal line $d$. Calciferous glands with welldeveloped diverticula in 11. Hearts in segments 7-11, nephridial bladders biscuit-shaped.

External characters. Holotype $132 \mathrm{~mm}$ long and $6 \mathrm{~mm}$ wide. Number of segments 134. Paratypes $113-121 \mathrm{~mm}$ long and 5-5.5 mm wide. Number of segments $153-157$. Pigmentation grey with slight reddish hints. Prostomium epilobous $1 / 2$ closed. First dorsal pore at the intersegmental furrow 8/9. Setae distantly standing. Setal arrangement behind clitellum: aa:ab:bc:cd:dd = 1.57:1.29:1.19:1:1.95. Male pores hardly seen on segment 15 . Nephridial pores just above setal line $b$. Clitellum on segments 25-30. Tubercula pubertatis on segments $1 / 2$ 25-1/2 29. Glandular tumescence on segments 10, 11, 13, 14, 26, 29, $30 a b$.

Internal characters. Septa 6/7-7/8 and 11/12 slightly thickened, 8/9-10/11 thickened, $12 / 13-14 / 15$ strongly thickened. Testes and funnels paired in segments $10-11$, covered by 


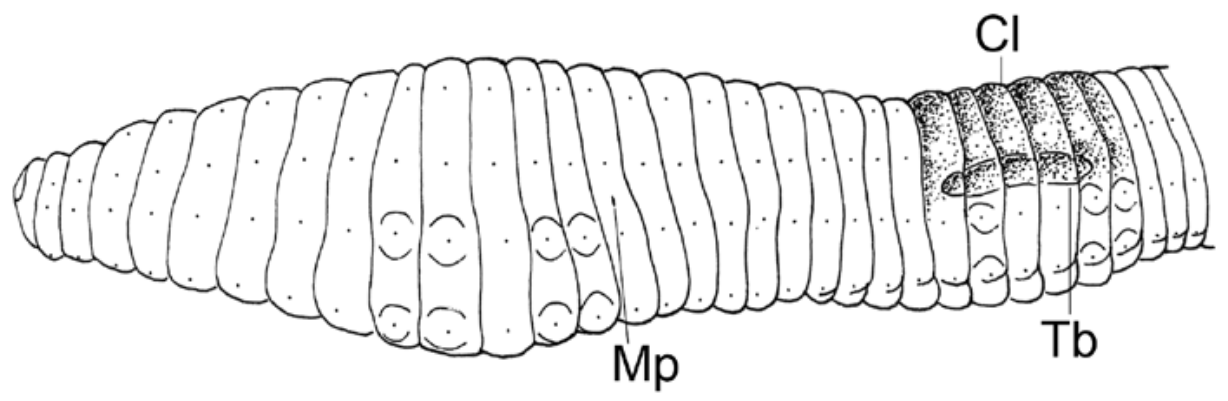

Fig. 1. Ventrolateral view of Dendrobaena cinerea $\mathrm{sp}$. $\mathrm{n}$. $\mathrm{Mp}=$ male pore, $\mathrm{Cl}=$ clitellum, $\mathrm{Tb}=$ tubercles

perioesophageal testis sac in segment 10-11. Four pairs of seminal vesicles in 9-12. Two pairs of spermathecae in 9/10, 10/11 with external openings in setal line $d$. Calciferous glands with well-developed diverticula in segment 11. Paired hearts in segments 7-11. with a pair of small extraoesophageal vessels in 12. Nephridial bladders biscuit-shaped.

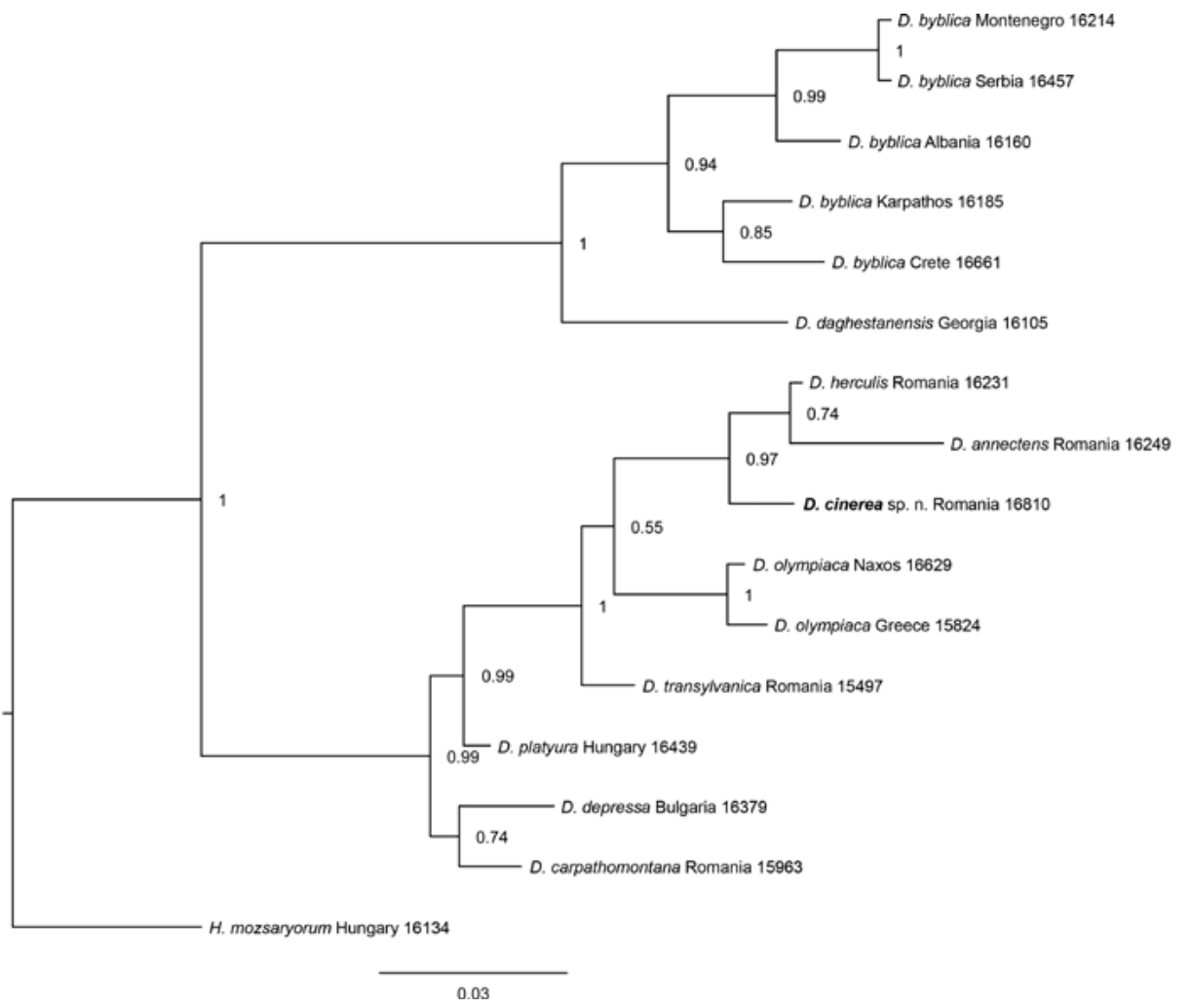

Fig. 2. Bayesian inference tree of $D$. cinerea sp. n. and the morphologically similar species, based on the ITS2 sequences. Numbers indicate the posterior probability values 
Table 3. Distinguishing characters of the species similar to $D$. cinerea sp. $\mathrm{n}$.

\begin{tabular}{|c|c|c|c|c|c|c|}
\hline Species & Clitellum & Tubercles & $\begin{array}{l}\text { Male } \\
\text { pore }\end{array}$ & Vesicles & $\begin{array}{l}\text { Sperma- } \\
\text { thecae }\end{array}$ & Colour \\
\hline $\begin{array}{l}\text { Dendrobaena } \\
\text { cinerea sp. n. }\end{array}$ & $25-30$ & $1 / 225-1 / 229$ & 15 & $9-12$ & $\begin{array}{c}\text { 9/10 } \\
10 / 11 d\end{array}$ & greyish \\
\hline $\begin{array}{l}\text { D. herculis Sze- } \\
\text { derjesi, Pop et } \\
\text { Csuzdi, } 2017\end{array}$ & $1 / 224-1 / 230$ & $26-28$ & 15 & $9-12$ & $\begin{array}{c}\text { 9/10, } \\
10 / 11 d\end{array}$ & $\begin{array}{l}\text { dark red- } \\
\text { violet }\end{array}$ \\
\hline $\begin{array}{l}\text { D. transylvanica } \\
\text { Szederjesi, Pop et } \\
\text { Csuzdi, } 2017\end{array}$ & $25-29$ & $26-28$ & 15 & $9-12$ & $\begin{array}{c}9 / 10 \\
10 / 11 d\end{array}$ & $\begin{array}{l}\text { dark red- } \\
\text { violet }\end{array}$ \\
\hline $\begin{array}{l}\text { D. annectens } \\
\text { (Rosa, 1895) }\end{array}$ & $\begin{array}{c}25-1 / 230 \\
30\end{array}$ & $26-28$ & 23 or 24 & $9-12$ & $\begin{array}{l}\text { 9/10, } \\
10 / 11 d\end{array}$ & $\begin{array}{l}\text { dark red- } \\
\text { violet }\end{array}$ \\
\hline $\begin{array}{l}\text { D. olympiaca } \\
\text { (Michaelsen, } \\
\text { 1902) }\end{array}$ & $25-30$ & $26-28$ & 15 & $9-12$ & $\begin{array}{c}\text { 9/10, } \\
10 / 11 d\end{array}$ & $\begin{array}{l}\text { dark red- } \\
\text { violet }\end{array}$ \\
\hline $\begin{array}{l}\text { D. platyura } \\
\text { (Fitzinger, 1833) }\end{array}$ & $\begin{array}{l}1 / 224 \\
25-30\end{array}$ & $25,26-29$ & 26 or 27 & $\begin{array}{l}9,11,12 \\
\text { or } 9-12\end{array}$ & $\begin{array}{l}\text { 9/10, } \\
10 / 11 d\end{array}$ & red-violet \\
\hline $\begin{array}{l}\text { D. depressa } \\
\text { (Rosa, 1893) }\end{array}$ & $\begin{array}{l}1 / 224 \\
25-30\end{array}$ & $\begin{array}{c}25,26-29 \\
30\end{array}$ & 26 & $\begin{array}{l}9,11,12 \\
\text { or } 9-12\end{array}$ & $\begin{array}{l}6 / 7,7 / 8 \\
8 / 9- \\
10 / 11 d\end{array}$ & $\begin{array}{l}\text { dark } \\
\text { grey with } \\
\text { reddish } \\
\text { iridescence }\end{array}$ \\
\hline $\begin{array}{l}\text { D. carpathomon- } \\
\text { tana (Szederjesi, } \\
\text { Pop et Csuzdi, } \\
\text { 2017) }\end{array}$ & $\begin{array}{c}1 / 224 \\
25-1 / 230 \\
30\end{array}$ & $1 / 225-1 / 230$ & 25 & 11,12 & $\begin{array}{l}6 / 7,7 / 8 \\
8 / 9- \\
10 / 11 d\end{array}$ & $\begin{array}{l}\text { dark } \\
\text { grey with } \\
\text { reddish } \\
\text { iridescence }\end{array}$ \\
\hline
\end{tabular}

Crop in segments 15-16, and gizzard in segments 17-18. Typhlosolis unilobous. The crosssection of the longitudinal muscle layer of pinnate type.

Remarks. According to the phylogenetic reconstruction based on the ITS2 sequences, the new species is placed into the clade of species bearing redviolet or greyish pigmentation and calciferous diverticula in segment 11 (Fig. 2). Dendrobaena sp. n. stands most closely to D. herculis Szederjesi, Pop et Csuzdi, 2017 and D. annectens (Rosa, 1895). Morphologically it most resembles D. depressa (Rosa, 1893) and D. carpathomontana (Szederjesi, Pop et Csuzdi, 2017) with its size and the clitellar organs, but differs from them in the position of the male pore and colouration. The most important morphological characteristics of the similar species are shown in Table 3. 


\section{Dendrobaena alpina alpina (Rosa, 1884)}

Allolobophora alpina Rosa, 1884: 28.

Dendrobaena alpina alpina: Pop et al. 2012: 63.

Material examined. HNHM/17233 6 ex., HNHM/17246 5 ex., Țarcu Mts, between

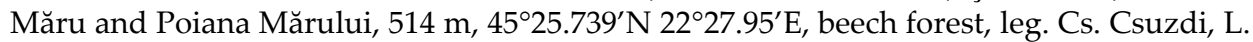
Dányi, V.V. Pop, T. Szederjesi, 09.11.2016. HNHM/17381 3 ex., Ţarcu Mts, Borlova, beech forest, 847 m, N45¹9.838’ E22²7.512', leg. Cs. Csuzdi, J. Kontschán, V.V. Pop.

\section{Dendrobaena alpina popi Šapkarev, 1971}

Dendrobaena alpina popi Šapkarev, 1971: 159. Pop et al. 2012: 63.

Material examined. HNHM/17203 10 ex., Parâng Mts, between Novaci and Rânca, $1132 \mathrm{~m}, 45^{\circ} 13.885^{\prime} \mathrm{N} 23^{\circ} 41.2141^{\prime} \mathrm{E}$, alpine pasture with scattered beech trees, leg. Cs. Csuzdi, L. Dányi, V.V. Pop, T. Szederjesi, 12.11.2016. HNHM/17219 3 ex., Vâlcan Mts, N of Vaidei, bank of Şuşita Verde, $425 \mathrm{~m}, 4^{\circ} 11.6738^{\prime} \mathrm{N} 23^{\circ} 16.1299^{\prime} \mathrm{E}$, beech forest, leg. Cs. Csuzdi, L. Dányi, V. V. Pop, T. Szederjesi, 11.11.2016. HNHM/17222 15 ex., Parâng Mts, stream valley NW of Novaci, $505 \mathrm{~m}, 45^{\circ} 11.5962^{\prime} \mathrm{N} 23^{\circ} 39.95^{\prime} \mathrm{E}$, chestnut forest patch, leg. Cs. Csuzdi, L. Dányi, V. V. Pop, T. Szederjesi, 13.11.2016.

\section{Dendrobaena annectens (Rosa, 1895)}

Allolobophora ganglbaueri var. annectens Rosa, 1895: 7.

Fitzingeria annectens: Pop et al. 2012: 64.

Dendrobaena annectens: Szederjesi et al. 2017: 508.

Material examined. HNHM/17197 6 ex., Parâng Mts, N of Novaci, bank of Gilort, $658 \mathrm{~m}, 45^{\circ} 13.388^{\prime} \mathrm{N} 23^{\circ} 40.22^{\prime} \mathrm{E}$, mixed forest, leg. Cs. Csuzdi, L. Dányi, V. V. Pop, T. Szederjesi, 11.11.2016. HNHM/17201 6 ex., Parâng Mts, stream valley NW of Novaci, 497 m, $45^{\circ} 11.8602^{\prime} \mathrm{N} 23^{\circ} 39.5161^{\prime} \mathrm{E}$, mixed forest with hornbeam, beech and alder, leg. Cs. Csuzdi, L. Dányi, V. V. Pop, T. Szederjesi, 13.11.2016. HNHM/17213 9 ex., HNHM/17223 3 ex., Parâng Mts, stream valley $\mathrm{NW}$ of Novaci, $505 \mathrm{~m}, 45^{\circ} 11.5962^{\prime} \mathrm{N} 23^{\circ} 39.95^{\prime} \mathrm{E}$, chestnut forest patch, leg. Cs. Csuzdi, L. Dányi, V. V. Pop, T. Szederjesi, 13.11.2016. HNHM/17226 4 ex., Vâlcan Mts, N of Vaidei, bank of Şuşita Verde, $452 \mathrm{~m}, 45^{\circ} 11.8473^{\prime} \mathrm{N} 23^{\circ} 16.0514^{\prime} \mathrm{E}$, beech forest, leg. Cs. Csuzdi, L. Dányi, V. V. Pop, T. Szederjesi, 11.11.2016. HNHM/17232 44 ex., Parâng Mts, stream valley NW of Novaci, $497 \mathrm{~m}, 45^{\circ} 11.8602^{\prime} \mathrm{N} 23^{\circ} 39.5161^{\prime} \mathrm{E}$, mixed forest with hornbeam, beech and alder, leg. Cs. Csuzdi, L. Dányi, V. V. Pop, T. Szederjesi, 13.11.2016.

\section{Dendrobaena attemsi (Michaelsen, 1902)}

Helodrilus (Dendrobaena) attemsi Michaelsen, 1902: 47.

Dendrobaena attemsi: Pop et al. 2012: 63. 
Material examined. HNHM/17206 1 ex., between Tismana and Peştişani, $182 \mathrm{~m}$, $45^{\circ} 2.62^{\prime} \mathrm{N} 22^{\circ} 59.49^{\prime} \mathrm{E}$, mixed hornbeam and oak forest, leg. Cs. Csuzdi, L. Dányi, V.V. Pop, T. Szederjesi, 11.11.2016.

\section{Dendrobaena carpathomontana (Szederjesi, Pop et Csuzdi, 2017)}

Octolasion montanum Černosvitov, 1932: 535.

Fitzingeria platyura montana: Pop et al. 2012: 64.

Dendrobaena carpathomontana: Szederjesi et al. 2017: 509.

Material examined. HNHM/17192 9 ex., Parâng Mts, N of Novaci, 675 m, 45ํ⒓0436’ N $23^{\circ} 41.5426^{\prime} \mathrm{E}$, pasture with alder and hawthorn, leg. Cs. Csuzdi, L. Dányi, V. V. Pop, T. Szederjesi, 12.11.2016.

\section{Dendrobaena herculis Szederjesi, Pop et Csuzdi, 2017}

Dendrobaena herculis Szederjesi, Pop et Csuzdi, 2017: 510.

Material examined. HNHM/17224 6 ex., Cerna Valley, NE of Băile Herculane, 208

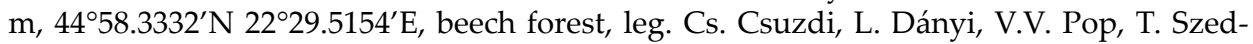
erjesi, 10.11.2016. HNHM/17243 3 ex., Țarcu Mts, between Măru and Poiana Mărului, 514 m, $45^{\circ} 25.739^{\prime} \mathrm{N} 22^{\circ} 27.95^{\prime} \mathrm{E}$, beech forest, leg. Cs. Csuzdi, L. Dányi, V.V. Pop, T. Szederjesi, 09.11.2016. HNHM/17247 4 ex., Ţarcu Mts, between Măru and Poiana Mărului, $440 \mathrm{~m}$, $45^{\circ} 25.97^{\prime} N 22^{\circ} 27.561^{\prime} E$, mixed beech forest, leg. Cs. Csuzdi, L. Dányi, V.V. Pop, T. Szederjesi, 10.11.2016. HNHM/17254 10 ex., Țarcu Mts, between Măru and Poiana Mărului, 635 $\mathrm{m}, 4^{\circ} 25.05^{\prime} \mathrm{N} 22^{\circ} 29.19^{\prime} \mathrm{E}$, young mixed beech forest, leg. Cs. Csuzdi, L. Dányi, V.V. Pop, T. Szederjesi, 09.11.2016.

\section{Dendrobaena octaedra (Savigny, 1826)}

Enterion octaedrum Savigny, 1826: 183.

Dendrobaena octaedra: Pop et al. 2012: 63.

Material examined. HNHM/17207 1 ex., between Tismana and Peştişani, $182 \mathrm{~m}$, $45^{\circ} 2.62^{\prime} \mathrm{N} 22^{\circ} 59.49^{\prime} \mathrm{E}$, mixed hornbeam and oak forest, leg. Cs. Csuzdi, L. Dányi, V. V. Pop, T. Szederjesi, 11.11.2016. HNHM/17365 1 ex., Argeș county, NW of Dâmbovițioara, along the road to Dâmbovițioara Cave, 920 m, N45.4464 E25.2207, leg. T. Asami, Z. Fehér, G. Katona, B. Páll-Gergely, 28.08.2018. HNHM/17368 1 ex., Dâmbovița county, 5 km NE of Glod, at the bridge of Valea Largă, 790 m, N45.2735 E25.5001, leg. T. Asami, Z. Fehér, G. Katona, B. Páll-Gergely, 27.08.2018. HNHM/17380 1 ex., Țarcu Mts, Borlova, beech forest, 847 m, N45⒚838' E22²7.512', leg. Cs. Csuzdi, J. Kontschán, V. V. Pop, 01.11.2007.

\section{Dendrobaena virgata Szederjesi, Pop et Csuzdi, 2014}

Dendrobaena virgata Szederjesi, Pop et Csuzdi 2014: 91. 
Material examined. HNHM/16809 1 ex., Țarcu Mts, spring and its outlet at Cuntu Meteorological Station, 1465 m, leg. T. Kovács, D. Murányi, G. Puskás, 09.06.2011. HNHM/17230 14 ex., Țarcu Mts, between Borlova and Muntele Mic, 495 m, 45 20.5163’ N $22^{\circ} 24.4343^{\prime}$ E, mixed beech forest, leg. Cs. Csuzdi, L. Dányi, V. V. Pop, T. Szederjesi, 10.11.2016. HNHM/17239 8 ex., Țarcu Mts, between Măru and Poiana Mărului, 443 m, $45^{\circ} 26.0775^{\prime} \mathrm{N} 22^{\circ} 27.4282^{\prime} \mathrm{E}$, mixed beech forest, leg. Cs. Csuzdi, L. Dányi, V.V. Pop, T. Szederjesi, 10.11.2016. HNHM/17244 2 ex., Țarcu Mts, between Măru and Poiana Mărului, $514 \mathrm{~m}, 4^{\circ} 25.739^{\prime} \mathrm{N} 22^{\circ} 27.95^{\prime} \mathrm{E}$, beech forest, leg. Cs. Csuzdi, L. Dányi, V. V. Pop, T. Szederjesi, 09.11.2016. HNHM/17248 7 ex., Țarcu Mts, between Măru and Poiana Mărului, $440 \mathrm{~m}, 45^{\circ} 25.97^{\prime} \mathrm{N} 22^{\circ} 27.561^{\prime} \mathrm{E}$, mixed beech forest, leg. Cs. Csuzdi, L. Dányi, V. V. Pop, T. Szederjesi, 10.11.2016. HNHM/17253 1 ex., Țarcu Mts, between Măru and Poiana Mărului, $635 \mathrm{~m}, 4^{\circ} 25.05^{\prime} \mathrm{N} 22^{\circ} 29.19^{\prime} \mathrm{E}$, young mixed beech forest, leg. Cs. Csuzdi, L. Dányi, V. V. Pop, T. Szederjesi, 09.11.2016. HNHM/17382 1 ex., Țarcu Mts, Borlova, beech forest, $847 \mathrm{~m}$, N45⒚838’ E22²7.512', leg. Cs. Csuzdi, J. Kontschán, V. V. Pop.

\section{Eisenia fetida (Savigny, 1826)}

Enterion fetidum Savigny, 1826: 182.

Eisenia fetida: Pop et al. 2012: 63.

Material examined. HNHM/17363 1 ex., Brașov county, Poiana Brașov, near Coliba Haiducilor, forest, 1070 m N45.5885 E25.554, leg. T. Asami, Z. Fehér, 29.08.2018.

\section{Eisenia lucens (Waga, 1857)}

Lumbricus lucens Waga, 1857: 161.

Eisenia lucens: Pop et al. 2012: 63.

Material examined. HNHM/17196 1 ex., Parâng Mts, between Novaci and Rânca, 722 $\mathrm{m}, 4^{\circ} 12.2333^{\prime} \mathrm{N} 23^{\circ} 41.8088^{\prime} \mathrm{E}$, beech forest, leg. Cs. Csuzdi, L. Dányi, V. V. Pop, T. Szederjesi, 12.11.2016. HNHM/17208 1 ex., between Tismana and Peştişani, $182 \mathrm{~m}, 45^{\circ} 2.62^{\prime} \mathrm{N}$ $22^{\circ} 59.49^{\prime} \mathrm{E}$, mixed hornbeam and oak forest, leg. Cs. Csuzdi, L. Dányi, V. V. Pop, T. Szederjesi, 11.11.2016. HNHM/17216 1 ex., Parâng Mts, stream valley NW of Novaci, 505 m, $45^{\circ} 11.5962^{\prime}$ N 2339.95’E, chestnut forest patch, leg. Cs. Csuzdi, L. Dányi, V. V. Pop, T. Szederjesi, 13.11.2016. HNHM/17245 4 ex., Țarcu Mts, between Măru and Poiana Mărului, 514 $\mathrm{m}, 4^{\circ} 25.739^{\prime} \mathrm{N} 22^{\circ} 27.95^{\prime} \mathrm{E}$, beech forest, leg. Cs. Csuzdi, L. Dányi, V. V. Pop, T. Szederjesi, 09.11.2016. HNHM/17250 2 ex., Țarcu Mts, between Măru and Poiana Mărului, 440 m, $45^{\circ} 25.97^{\prime} \mathrm{N} 22^{\circ} 27.561^{\prime} \mathrm{E}$, mixed beech forest, leg. Cs. Csuzdi, L. Dányi, V. V. Pop, T. Szederjesi, 10.11.2016. HNHM/17255 3 ex., Țarcu Mts, between Măru and Poiana Mărului, 635 $\mathrm{m}, 45^{\circ} 25.05^{\prime} \mathrm{N} 22^{\circ} 29.19^{\prime} \mathrm{E}$, young mixed beech forest, leg. Cs. Csuzdi, L. Dányi, V. V. Pop, T. Szederjesi, 09.11.2016. HNHM/17364 1 ex., Argeș county, NW of Dâmbovițioara, along the road to Dâmbovițioara Cave, 920 m, N45.4464 E25.2207, leg. T. Asami, Z. Fehér, G. Katona, B. Páll-Gergely, 28.08.2018. HNHM/17366 1 ex., Argeș county, E of Podu Dâmboviței, Valea Cheii, $4.5 \mathrm{~km}$ from the junction in the village, $910 \mathrm{~m}, \mathrm{~N} 45.4037$ E25.2541, leg. T. Asami, Z. Fehér, G. Katona, B. Páll-Gergely, 28.08.2018. 


\section{Eiseniella tetraedra (Savigny, 1826)}

Enterion tetraedrum Savigny, 1826: 184.

Eiseniella tetraedra: Pop et al. 2012: 63.

Material examined. HNHM/17227 1 ex., Vâlcan Mts, N of Vaidei, bank of Şuşita Verde, $452 \mathrm{~m}, 45^{\circ} 11.8473^{\prime} \mathrm{N} 23^{\circ} 16.0514^{\prime} \mathrm{E}$, beech forest, leg. Cs. Csuzdi, L. Dányi, V. V. Pop, T. Szederjesi, 11.11.2016. HNHM/17369 1 ex., Dâmbovița county, 5 km NE of Glod, at the bridge of Valea Largă, 790 m, N45.2735 E25.5001, leg. T. Asami, Z. Fehér, G. Katona, B. Páll-Gergely, 27.08.2018.

\section{Lumbricus polyphemus (Fitzinger, 1833)}

Enterion polyphemus Fitzinger, 1833: 552.

Lumbricus polyphemus: Pop et al. 2012: 64.

Material examined. HNHM/17211 5 ex., between Borlova and Baia de Aramă, 216 m, $45^{\circ} 0.4919^{\prime} \mathrm{N} 22^{\circ} 48.8658^{\prime} \mathrm{E}$, mixed hornbeam and oak forest with beech and chestnut, leg. Cs. Csuzdi, L. Dányi, V. V. Pop, T. Szederjesi, 11.11.2016. HNHM/17228 2 ex., Țarcu Mts, between Borlova and Muntele Mic, $495 \mathrm{~m}, 45^{\circ} 20.5163^{\prime} \mathrm{N} 22^{\circ} 24.4343^{\prime} \mathrm{E}$, mixed beech forest, leg. Cs. Csuzdi, L. Dányi, V. V. Pop, T. Szederjesi, 10.11.2016. HNHM/17238 4 ex., Țarcu Mts, between Măru and Poiana Mărului, 443 m, 45²6.0775’ N 22 27.4282'E, mixed beech forest, leg. Cs. Csuzdi, L. Dányi, V. V. Pop, T. Szederjesi, 10.11.2016. HNHM/17249 1 ex., Țarcu Mts, between Măru and Poiana Mărului, 440 m, 45²5.97' N 22 $27.561^{\prime} \mathrm{E}$, mixed beech forest, leg. Cs. Csuzdi, L. Dányi, V. V. Pop, T. Szederjesi, 10.11.2016.

\section{Lumbricus rubellus Hoffmeister, 1843}

Lumbricus rubellus Hoffmeister, 1843: 187. Pop et al. 2012: 64.

Material examined. HNHM/17236 1 ex., Țarcu Mts, S of Măru, 399 m, 45⒉ $27.6147^{\prime} \mathrm{N}$ $22^{\circ} 26.7447^{\prime} E$, pasture, leg. Cs. Csuzdi, L. Dányi, V.V. Pop, T. Szederjesi, 10.11.2016.

\section{Lumbricus terrestris Linnaeus, 1758}

Lumbricus terrestris Linnaeus, 1758: 647. Pop et al. 2012: 64.

Material examined. HNHM/17214 4 ex., Parâng Mts, stream valley NW of Novaci, $505 \mathrm{~m}, 45^{\circ} 11.5962^{\prime} \mathrm{N} 23^{\circ} 39.95^{\prime} \mathrm{E}$, chestnut forest patch, leg. Cs. Csuzdi, L. Dányi, V. V. Pop, T. Szederjesi, 13.11.2016. HNHM/17362 1 ex., Brașov county, Poiana Brașov, near Coliba Haiducilor, forest, 1070 m N45.5885 E25.554, leg. T. Asami, Z. Fehér, 29.08.2018. 


\section{Octodrilus banaticus Szederjesi, Pop et Csuzdi sp. n.} (Fig. 3)

Holotype. HNHM/17361 Țarcu Mts, between Borlova and Muntele Mic, $495 \mathrm{~m}$, $45^{\circ} 20.5163^{\prime} \mathrm{N} 22^{\circ} 24.4343^{\prime} \mathrm{E}$, mixed beech forest, leg. Cs. Csuzdi, L. Dányi, V. V. Pop, T. Szederjesi, 10.11.2016. Paratypes. HNHM/17231 1 ex. praeadult +6 ex. juvenile, locality and date same as that of the Holotype. HNHM/17240 1 ex. subadult +2 ex. praeadult +1 ex. juvenile, Țarcu Mts, between Măru and Poiana Mărului, 443 m, 4526.0775'N 22²7.4282'E, mixed beech forest, leg. Cs. Csuzdi, L. Dányi, V. V. Pop, T. Szederjesi, 10.11.2016. region.

Etymology. The name refers to the geographic region of the type locality, the Banat

Diagnosis. Length 145-171 mm, diameter 6-7 mm, setae distantly standing. Pigmentation greyish. First dorsal pore in 11/12. Clitellum on $1 / 229-1 / 239$, $1 / 240$, tubercles on $30-1 / 239,1 / 240$. Male pores on segment 15 . Nephridial pores aligned above setal line $b$. Three pairs of seminal vesicles in $9,10,12$; spermathecae 7 (6-8) pairs in 5/6-11/12 (12/13) in setal line $d$. Calciferous glands with well-developed diverticula in 10 . Hearts in segments 7-11, nephridial bladders ocarina-shaped.

External characters. Holotype $145 \mathrm{~mm}$ long and $7 \mathrm{~mm}$ wide. Number of segments 184. Paratypes 146-171 mm long and 6-6.5 mm wide. Number of segments 180-223. Pigmentation greyish. Prostomium epilobous $1 / 3$ closed. First dorsal pore at the intersegmental furrow 11/12. Setae distantly standing. Setal arrangement behind clitellum: aa:ab:bc: $\mathrm{cd}: \mathrm{dd}=3.38: 2.13: 1.22: 1: 5,07$. Male pores on segment 15, small. Nephridial pores aligned above setal line $b$. Clitellum on segments $1 / 229-1 / 239,1 / 240$. Tubercula pubertatis on segments $30-1 / 239,1 / 240$.

Internal characters. Septa 6/7-8/9 thickened, 12/13-14/15 strongly. Testes and funnels paired in segments $10-11$, covered by oesophageal testis sacs in segment 10 . The dissepiments of 10/11 and 11/12 merged together, completely enclosing the male funnels of 11. Three pairs of seminal vesicles in $9,10,12$. Usually seven (sometimes 6 or 8 ) pairs of spermathecae in 5/6-11/12 with external openings in setal line $d$. In some specimens asymmetrically missing from $5 / 6$ or extra pair present in $12 / 13$. Calciferous glands with

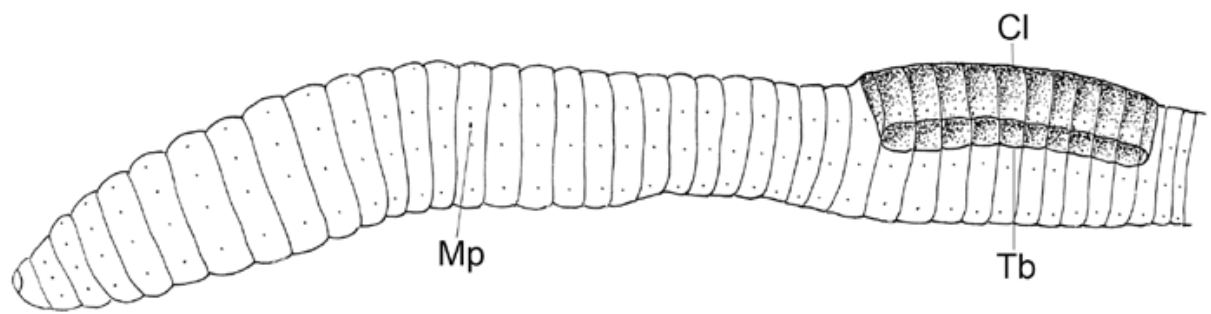

Fig. 3. Ventrolateral view of Octodrilus banaticus sp. $\mathrm{n}$. $\mathrm{Mp}=$ male pore, $\mathrm{Cl}=$ clitellum, $\mathrm{Tb}=$ tubercles 
Table 4. Distinguishing characters of the species similar to Oc. banaticus sp. n.

\begin{tabular}{|c|c|c|c|c|c|c|}
\hline Species & Clitellum & Tubercles & $\begin{array}{l}\text { Vesi- } \\
\text { cles }\end{array}$ & $\begin{array}{l}\text { Sperma- } \\
\text { thecae }\end{array}$ & $\begin{array}{l}\text { Testic } \\
\text { sac }\end{array}$ & $\begin{array}{c}\text { Nephri- } \\
\text { dial } \\
\text { pores }\end{array}$ \\
\hline Oc. banaticus sp. n. & $\begin{array}{c}1 / 229-1 / 239 \\
1 / 240\end{array}$ & $\begin{array}{c}30-1 / 239 \\
1 / 240\end{array}$ & $\begin{array}{c}9,10 \\
12\end{array}$ & $\begin{array}{l}7(5 / 6- \\
11 / 12)\end{array}$ & 10 oeso & $\begin{array}{l}\text { aligned } \\
\text { in } b\end{array}$ \\
\hline Oc. aporus Pop, 1989 & $1 / 229,30-40$ & $30-40$ & $9-12$ & $\begin{array}{l}6(5 / 6- \\
10 / 11)\end{array}$ & $\begin{array}{c}\text { 10, } 11 \\
\text { perioeso }\end{array}$ & irregular \\
\hline $\begin{array}{l}\text { Oc. hemiandrus } \\
\text { (Cognetti, 1901) }\end{array}$ & $28-36,37$ & $28-38,39$ & $\begin{array}{c}9,10 \\
12\end{array}$ & $\begin{array}{l}7(6 / 7- \\
12 / 13)\end{array}$ & 11 oeso & irregular \\
\hline $\begin{array}{l}\text { Oc. velebiticus } \\
\text { Mršić, } 1991\end{array}$ & $1 / 229-39$ & $30-39$ & $9-12$ & $\begin{array}{l}7(6 / 7- \\
12 / 13)\end{array}$ & $\begin{array}{c}10,11 \\
\text { oeso }\end{array}$ & irregular \\
\hline $\begin{array}{l}\text { Oc. complanatus } \\
\text { (Dugès, 1828) }\end{array}$ & $28,29-37$ & $30-39,40$ & $9-12$ & $\begin{array}{l}7(6 / 7- \\
12 / 13)\end{array}$ & $\begin{array}{c}10,11 \\
\text { oeso }\end{array}$ & irregular \\
\hline $\begin{array}{l}\text { Oc. vallorus (Bal- } \\
\text { dasseroni, 1920) }\end{array}$ & $30-39$ & $29,30-39$ & $9-12$ & $\begin{array}{l}6(6 / 7- \\
11 / 12)\end{array}$ & $\begin{array}{c}10,11 \\
\text { perioeso }\end{array}$ & irregular \\
\hline $\begin{array}{l}\text { Oc. mimus } \\
\text { (Rosa, 1889) }\end{array}$ & $\begin{array}{c}28,29-40 \\
41\end{array}$ & $\begin{array}{c}28,29-40, \\
41\end{array}$ & $9-12$ & $\begin{array}{l}7(6 / 7- \\
12 / 13)\end{array}$ & $\begin{array}{c}\text { 10, } 11 \\
\text { perioeso }\end{array}$ & irregular \\
\hline $\begin{array}{l}\text { Oc. pseudocomplana- } \\
\text { tus (Omodeo, 1962) }\end{array}$ & $\begin{array}{c}1 / \mathrm{n} 29 \\
30-37,1 / 238\end{array}$ & $\begin{array}{c}1 / \mathrm{n} 29 \\
30-39,40\end{array}$ & $9-12$ & $\begin{array}{l}7(6 / 7- \\
12 / 13)\end{array}$ & $\begin{array}{c}10,11 \\
\text { perioeso }\end{array}$ & $\begin{array}{l}\text { aligned } \\
\text { in } b\end{array}$ \\
\hline
\end{tabular}

well-developed diverticula in segment 10. Paired hearts in segments 7-11. with a pair of small extraoesophageal vessels in 12. Nephridial bladders ocarina-shaped. Crop in segments 15-16, and gizzard in segments 17-18. Typhlosolis lamelliform. The cross-section of the longitudinal muscle layer of pinnate type.

Remarks. Morphologically the new species is most close to Oc. aporus Pop, 1989 (cl: $1 \frac{1}{2} 29,30-40$ and t: 30-40), but differs from it with the number of vesicles ( $3 v s .4$ pairs), spermathecae ( $7 v s .6$ pairs) and the type of the testic sacs (oesophageal vs. perioesophageal). Oc. banaticus sp. n. is similar to Oc. hemiandrus (Cognetti, 1901) with the lack of vesicles in segment 11, the number of spermathecae and the oesophageal testis sacs, but differs with the clitellar organs (cl: 28-36, 37, t: 28-38, 39). It is close to Oc. velebiticus Mršić, 1991 with its clitellum and tubercles (cl: 1/2 29-39, t: 30-39), but differs with the number of vesicles ( 3 vs. 4 pairs). The tubercles of Oc. complanatus (Dugès, $1828)$ are quite similar $(30-39,40)$ but its clitellum is shorter $(28,29-37)$ and has 4 pairs of vesicles. Oc. vallorus (Baldasseroni, 1920) (cl: 30-39, t: 29, 30-39) and Oc. mimus (Rosa, 1889) (cl: 28, 29-40, $41 \mathrm{t:} 28,29-40,41)$ are similar with their clitellar organs, but differ with the number of spermathecae (6 and 6-7 pairs), the number of vesicles (4 pairs) and the periesophageal testis sacs. Oc. pseudocomplanatus (Omodeo, 1962) is very similar to the new species with its tubercles $(1 / \mathrm{n} 29,30-39,40)$ and the aligned nephridiopores, but differs with its shorter clitellum $(1 / \mathrm{n} 29,30-37,1 / 238)$, reddish colour, the perioesophageal 
testis sacs and the four pairs of vesicles. The distinguishing characters of the similar species are shown in Table 4 .

\section{Octodrilus compromissus compromissus Zicsi et Pop, 1984}

Octodrilus compromissus Zicsi et Pop, 1984: 245.

Octodrilus compromissus compromissus: Pop et al. 2012: 64.

Material examined. HNHM/17218 4 ex., Vâlcan Mts, N of Vaidei, bank of Şuşita Verde, $425 \mathrm{~m}, 45^{\circ} 11.6738^{\prime} \mathrm{N} 23^{\circ} 16.1299^{\prime} \mathrm{E}$, beech forest, leg. Cs. Csuzdi, L. Dányi, V. V. Pop, T. Szederjesi, 11.11.2016. HNHM/17251 2 ex., Țarcu Mts, between Măru and Poiana Mărului, 440 m, $45^{\circ} 25.97^{\prime} N 22^{\circ} 27.561^{\prime}$ E, mixed beech forest, leg. Cs. Csuzdi, L. Dányi, V. V. Pop, T. Szederjesi, 10.11.2016.

\section{Octodrilus exacystis exacystis (Rosa, 1896)}

Allolobophora exacystis Rosa, 1896: 3.

Octodrilus exacystis exacystis: Pop et al. 2012: 64.

Material examined. HNHM/17241 9 ex., Țarcu Mts, between Măru and Poiana Mărului, $443 \mathrm{~m}, 45^{\circ} 26.0775^{\prime} \mathrm{N} 22^{\circ} 27.4282^{\prime} \mathrm{E}$, mixed beech forest, leg. Cs. Csuzdi, L. Dányi, V. V. Pop, T. Szederjesi, 10.11.2016. HNHM/17252 9 ex., Țarcu Mts, between Măru and Poiana

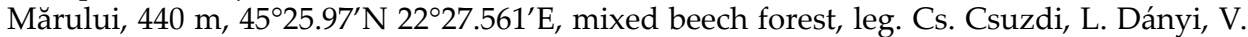
V. Pop, T. Szederjesi, 10.11.2016. HNHM/17256 2 ex., Țarcu Mts, between Măru and Poiana Mărului, $635 \mathrm{~m}, 45^{\circ} 25.05^{\prime} \mathrm{N} 22^{\circ} 29.19^{\prime} \mathrm{E}$, young mixed beech forest, leg. Cs. Csuzdi, L. Dányi, V. V. Pop, T. Szederjesi, 09.11.2016.

\section{Octodrilus transpadanus (Rosa, 1884)}

Allolobophora transpadana Rosa, 1884: 45.

Octodrilus transpadanus: Pop et al. 2012: 64.

Material examined. HNHM/17205 4 ex., between Tismana and Peştişani, $182 \mathrm{~m}$, $45^{\circ} 2.62^{\prime} \mathrm{N} 22^{\circ} 59.49^{\prime} \mathrm{E}$, mixed hornbeam and oak forest, leg. Cs. Csuzdi, L. Dányi, V. V. Pop, T. Szederjesi, 11.11.2016.

\section{Octolasion lacteum (Örley, 1881)}

Lumbricus terrestris var. lacteus Örley, 1881: 584.

Octolasion lacteum: Pop et al. 2012: 64.

Material examined. HNHM/17199 2 ex., Parâng Mts, N of Novaci, bank of Gilort,

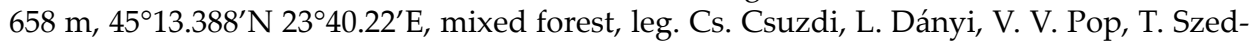
erjesi, 11.11.2016. HNHM/17202 2 ex., Parâng Mts, N of Baia de Fier, bank of Galbenul, 654

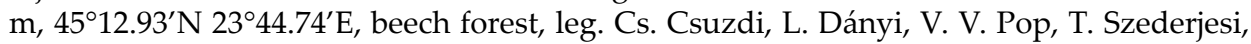
12.11.2016. HNHM/17221 1 ex., Vâlcan Mts, N of Vaidei, bank of Şuşita Verde, 425 m, 
$45^{\circ} 11.6738^{\prime} \mathrm{N} 23^{\circ} 16.1299^{\prime} \mathrm{E}$, beech forest, leg. Cs. Csuzdi, L. Dányi, V. V. Pop, T. Szederjesi, 11.11.2016. HNHM/17225 1 ex., Cerna Valley, NE of Băile Herculane, 208 m, 44 58.3332'N $22^{\circ} 29.5154^{\prime}$ E, beech forest, leg. Cs. Csuzdi, L. Dányi, V. V. Pop, T. Szederjesi, 10.11.2016. HNHM/17367 1 ex., Brașov county, 1.2 km W of Poiana Brașov, Pârâul Poiana valley, forest, 960 m, N45.5953 E25.5367, leg. T. Asami, Z. Fehér, 29.08.2018.

\section{DISCUSSION}

The present research resulted in reporting altogether 26 earthworm species from different parts of the Southern Carpathians, among them two species new to science. With all these, the number of earthworm species and subspecies present in Romania is now 80.

Unfortunately only the ITS2 region was available for the molecular study of $D$. cinerea sp. n., but our results clearly show the separateness of this species. It fits well the clade of some Carpatho-Balkanic Dendrobaena species revealed by SzEDERJEsi et al. (2017) both morphologically and genetically. The presence of a certain kind of pigmentation, calciferous diverticula in segment 11 and the similar position of the clitellar organs make this group very uniform.

Morphologically Oc. banaticus sp. $\mathrm{n}$. is quite similar to several species with different distribution, e.g. the Dacian Oc. aporus, the Circum-Mediterranean Oc. complanatus and also the West Balkanic Octodrilus species. Additional molecular studies should be carried out to clarify its exact phylogenetic connections.

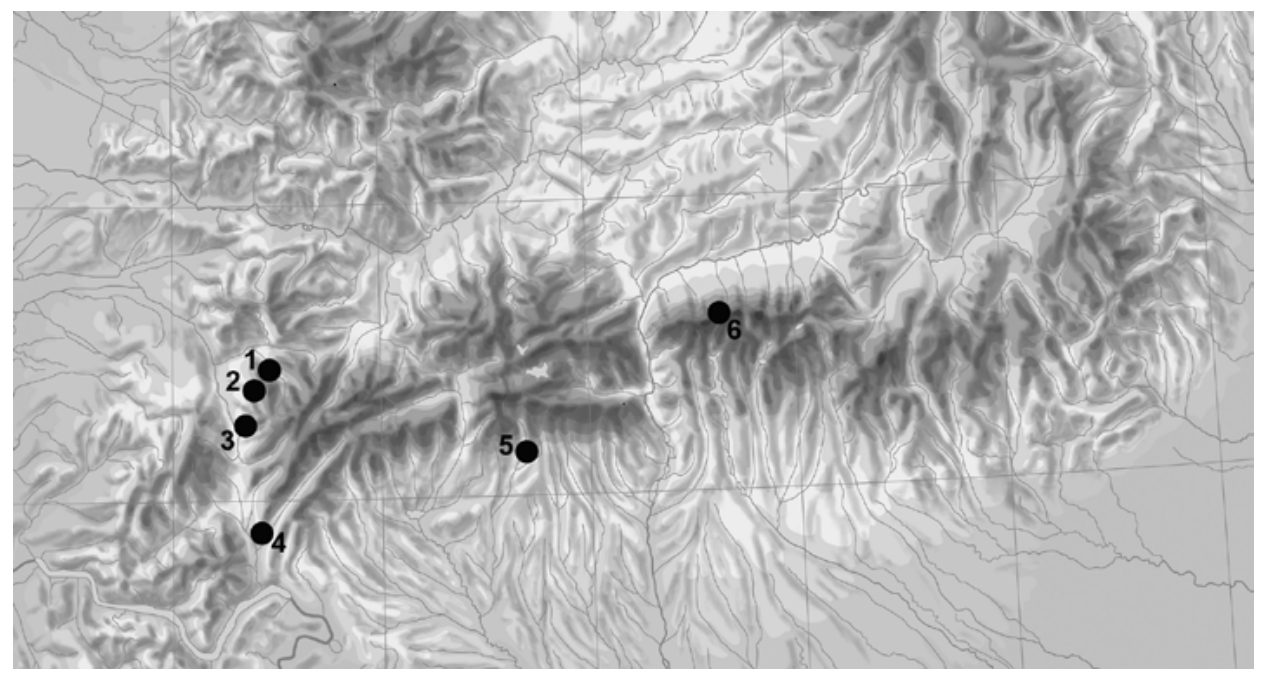

Fig. 4. Type localities of the Southern Carpathian endemic earthworm species. $1=D$. cinerea sp. n. and D. virgata, $2=$ Oc. banaticus sp. n., $3=C$. getica, $4=D$. herculis, $5=A$. pannonica (exact type locality is not known), $6=D$. annectens 
A. pannonica (Cognetti, 1906) has been resurrected from synonymy of $A$. mehadiensis mehadiensis and reinstated as a separate species due to its unique characteristics. At the same time, morphologically this species is most close to the $A$. mehadiensis subspecies however, its general appearance is reminiscent of an A. sturanyi dacica specimen.

Several molecular phylogenetic analyses (Pop et al. 2005, Domínguez et al. 2015, SzEDERJEsi et al. 2016) revealed a well-defined Carpatho-Balkanic clade consisting of the A. sturanyi subspecies, A. gestroides, A. gestroi, A. zicsica, the A. mehadiensis subspecies and the Cernosvitovia species. These endogeic taxa share common characteristics such as a long clitellum (usually more than 10 segments) and tubercles, large segment number, strongly thickened dissepiments and the occurrence of secondary or tertiary annulations. On morphological basis $A$. pannonica also belongs to this group, however further molecular phylogenetic analysis is needed to determine the exact phylogenetic position of this species.

The Southern Carpathians, and within it the Tarcu Mts and the Cerna valley has several endemic earthworm species, such as Dendrobaena annectens, D. herculis, D. virgata, Cernosvitovia getica and the three species discussed above (Fig. 4). The complex geohistory certainly played a significant role in the evolution of such high number of endemics in this region. From the Miocene, as the Carpathian arch emerged, the repeated regressions and transgressions of the Paratethys created an archipelago-like formation of the Carpathian palaeo-islands (Hurdu et al. 2016, Harzhauser \& Piller 2007). The Pleistocene glacials affected the Carpathians only patchily, mostly concerning on the highest elevations, which led to the appearance of several refugial areas (Schmitt 2009, Mráz \& Ronikier 2016). Besides, the Banat Mountains region and the Cerna valley seems to be an important biogeographical corridor and a meeting-place of different Mediterranean and Carpathian elements, as highlighted by CAMERon et al. (2016) with investigation of the forest land snail fauna. In case of earthworms, several East Balkanic and Mediterranean species, eg. A. robusta, C. rebeli, D. byblica, reach the Southern Carpathians through this region (CsuzDi et al. 2011). The Balkanic connections are also emphasised by the phylogenetic relationship between the Southern Carpathian D. annectens, D. herculis, D. cinerea sp. n. and the Balkanic D. olympiaca.

Acknowledgements - The study was supported by the Hungarian Scientific Research Fund (OTKA K100369) and the SYNTHESYS3 project to T. Szederjesi (AT-TAF 6851) which is financed by the European Community Research Infrastructure Action under the FP7 "Capacities" Programme. We are grateful for the work of all collectors. 


\section{REFERENCES}

Benson, D. A., Karsch-Mizrachi, I., Clark, K., Lipman, D. J., Ostell, J. \& Sayers, E. W. (2012): Genbank. - Nucleid Acids Research 40(Database issue): D48-D53. https://doi .org/10.1093/nar/gks1195

Cameron, R. A. D., Pokryszko, B. M., Gheoca, V. \& Horsák, M. (2016): At the Central European-Balkan transition: forest land snail fauna of the Banat contrasted with those of the Carpathian chain. - Biological Journal of the Linnean Society 119(3): 560-570. https:// doi.org/10.1111/bij.12498

Černosvitov, L. (1932): Die Oligochaetenfauna der Karpathen. II. Die Lumbriciden und ihre Verbreitung. - Zoologische Jahrbücher Systematik 62: 525-546.

Cognetti, M. (1906): Nuovi dati sui Lumbricidi dell'Europa orientale. - Bollettino dei Musei di zoologia ed anatomia comparata della $R$. Universitá di Torino 21(257): 1-18.

Csuzdi, Cs., Pop, V. V. \& Pop, A. A. (2011): The earthworm fauna of the Carpathian Basin with new records and description of three new species (Oligochaeta: Lumbricidae). Zoologischer Anzeiger 250: 2-18. https://doi.org/10.1016/j.jcz.2010.10.001

Csuzdi, Cs., Chang, C-H., Pavlíček, T., Szederjesi, T., Esopi, D. \& Szlávecz, K. (2017): Molecular phylogeny and systematics of native North American lumbricid earthworms (Clitellata: Megadrili). - Plos One 12(8): e0181504. https://doi.org/10.1371/journal .pone.0181504

Domínguez, J., Aira, M., Breinholt, J. W., Stojanović, M., James, S. W. \& Pérez-Losada, M. (2015): Underground evolution: New roots for the old tree of lumbricid earthworms. - Molecular Phylogenetics E Evolution 83: 7-19. https://doi.org/10.1016/j.ympev.2014.10 .024

Fitzinger, L. (1833): Beobachtungen über die Lumbrici. - Isis 4: 549-553.

Harzhauser, M. \& Piller, W. E. (2007): Benchmark data of a changing sea - Palaeogeography, palaeobiogeography and events in the Central Paratethys during the Miocene. - Palaeogeography, Palaeoclimatology, Palaeoecology 253: 8-31.

Hoffmeister, W. (1843): Beitrag zur Kenntnis deutscher Landanneliden. - Archiv für Naturgeschichte 91: 183-198.

Hurdu, B-I., Escalante, T., Puşcaş, M., Novikoff, A., Bartha, L. \& Zimmermann, N. E. (2016): Exploring the different facets of plant endemism int he South-Eastern Carpathians: a manifold approach fot the determination of biotic elements, centres and areas of endemism. - Biological Journal of the Linnean Society 119(3): 649-672. https:// doi.org/10.1111/bij.12902

KatoH, K. \& STANDLEY, D. M. (2013): MAFFT multiple sequence alignment software version 7: improvements in performance and usability. - Molecular Biology and Evolution 30: 772-780. https://doi.org/10.1093/molbev/mst010

Linnaeus, C. (1758): Systema Naturae per Regna tria Naturae, secundum Classes, Ordines, Genera, Species, cum Characteribus, Differentiatiis, Synonymis, Locis. 10th ed., Vol. 1. - Laurentii Salvii, Holmiae, 824 pp.

Michaelsen, W. (1891): Oligochaeten des Naturhistorischen Museums in Hamburg IV. Jahrbuch der Hamburgischen Wissenschaftlichen Anstalten 8: 1-42.

Michaelsen, W. (1902): Neue Oligochaeten und neue Fundorte altbekannter. - Mitteilungen aus dem Naturhistorischen Museum in Hamburg 19: 3-53.

Michaelsen, W. (1903): Die geographische Verbreitung der Oligochaeten. - Friedländer \& Sohn, Berlin, $186 \mathrm{pp}$. 
MrÁz, P. \& Ronikier, M. (2016): Biogeography of the Carpathians: evolutionary and spatial facets of biodiversity. - Biological Journal of the Linnean Society 119(3): 528-559. https:// doi.org/10.1111/bij.12918

Mršić, N. (1991): Monograph on earthworms (Lumbricidae) of the Balkans I-II. - Slovenska Akademija Znanosti in Umetnosti, Ljubljana, 757 pp.

ÖRLEY, L. (1881): A magyarországi Oligochaeták faunája. I. Terricolae. - Mathematikai és Természettudományi Közlemények 16: 562-611.

ÖRLEY, L. (1885): A palearktikus övben élő Terrikoláknak revíziója és elterjedése. Értekezések a Természettudományok Köréból 15: 1-34.

Pop, A. A., Csuzdi, Cs., Wink, M. \& Pop, V. V. (2005): An attempt to reconstruct the molecular phylogeny of the genus Allolobophora Eisen, 1874 (sensu lato, Pop, 1941) using 16S rDNA and COI sequences (Oligochaeta, Lumbricidae). Pp. 155-164. In: Pop, V. V. \& Pop, A. A. (eds): Advances in Earthworm Taxonomy II. - Cluj University Press, Cluj-Napoca.

Pop, V. (1948): Allolobophora mehadiensis Rosa var. boscaiui, une nouvelle variété de Lumbricide et ses affinités. - Buletinul Societății de Ştiinţe din Cluj 10: 104-109.

Pop, V. (1949): Lumbricidele din România. - Analele Academiei Republicii Populare Române Secţiunea de Ştiinţe Geologice, Geografice şi Biologie 1(9): 383-505.

Pop, V. (1964): Noi date faunistice şi sistematice asupra lumbricidelor din România. - Studia Universitatis Babes-Bolyai, series Biologia 2: 107-116.

Pop, V. V., Pop, A. A. \& Csuzdi, Cs. (2012): An annotated checklist of the Romanian earthworm fauna (Oligochaeta, Lumbricidae). - Zoology in the Middle East 4: 59-70.

Rambaut, A. (2012): FigTree version 1.4.0. - Available from: http://tree.bio.ed.ac.uk /software/figtree/

RAw, F. (1959): Estimating earthworm populations by using formalin. - Nature 184: 16611662.

Ronquist, F., Teslenko, M., van der Mark, P., Ayres, D. L., Darling, A., Höhna, S., LarGet, B., Liu, L., Suchard, M. A. \& Huelsenbeck, J. P. (2012): MrBayes 3.2: efficient Bayesian phylogenetic inference and model choice across a large model space. - Systematic Biology 61: 539-542. https://doi.org/10.1093/sysbio/sys029

Rosa, D. (1884): Lumbricidi del Piemonte. - UnioneTipografico-Editrice, Torino, 54 pp.

Rosa, D. (1895): Nuovi lombrichi dell'Europa orientale. - Bollettino dei Musei di Zoologia ed Anatomia comparata della $R$. Universitá di Torino 10(21): 1-8.

RosA, D. (1896): Allobophora tigrina ed A. exacystis n. sp. - Bollettino dei Musei di Zoologia ed Anatomia comparata della R. Universitá di Torino 11(246): 1-4.

RosA, D. (1897): Nuovi lombrichi dell'Europa orientale. (Seconda serie). - Bollettino dei Musei di Zoologia ed Anatomia comparata della R. Universitá di Torino 12(269): 1-5.

ŠApKAREv, J. (1971): Neue Regenwürmer (Oligochaeta: Lumbricidae) aus Mazedonien. Fragmenta Balcanica Skopje 8(18): 149-164.

Savigny, J. C. (1826): Analyse d'un memoire sur les Lombrics par Cuvier. In: Cuvier, G.: Analyse des travaux de l'Academie royale des Sciences, pendant l'année 1821, partie physique. - Mémoires de l'Académie des Sciences de l'Institut de France Paris 5: 176-184.

Sснмітт, T. (2009): Biogeographical and evolutionary importance of the European high mountain systems. - Frontiers in Zoology 6: 9. https://doi.org/10.1186/1742-9994-6-9

Schwarz, G. E. (1978): Estimating the dimension of a model. - The Annals of Statistics 6(2): 461-464. 
Szederjesi, T., Pop, V. V. \& Csuzdi, Cs. (2014): New and little known earthworm species from peripheral areas of the Romanian Carpathians (Oligochaeta, Lumbricidae). Acta Zoologica Academiae Scientiarum Hungaricae 60(2): 85-107.

Szederjesi, T., Pop, V. V., Márton, O., Krízsik, V. \& Csuzdi, Cs. (2016): The Allolobophora sturanyi species group revisited: Integrated taxonomy and new taxa (Clitellata: Megadrili). - Opuscula Zoologica, Budapest 47(1): 87-92. https://doi.org/10.18348 /opzool.2016.1.87

Szederjesi, T., Pop, V. V., Pavlíček, T., Márton, O., Krízsik, V. \& Csuzdi, Cs. (2017): Integrated taxonomy reveals multiple species in the Dendrobaena byblica (Rosa, 1893) complex (Oligochaeta, Lumbricidae). - Zoological Journal of the Linnean Society 182(3): 500-516. https://doi.org/10.1093/zoolinnean/zlx049

WAGA, A. (1857): Sprawozdanie z podróży naturalistów odbytej w r. 1854 do Ojcowa. - Biblioteka warszawska 2: 161-227.

Zıcsı, A. \& Pop, V. V. (1984): Neue Regenwürmer aus Rumänien (Oligochaeta: Lumbricidae). - Acta Zoologica Academiae Scientiarum Hungaricae 30: 241-248.

Received January 24, 2019, accepted April 3, 2019, published May 31, 2019 